\title{
Integrating LFG's binding theory with PCDRT
}

\author{
Mary Dalrymple ${ }^{1}$, Dag T. T. Haug ${ }^{2}$, and John J. Lowe ${ }^{1}$ \\ ${ }^{1}$ Faculty of Linguistics, Philology and Phonetics, University of Oxford \\ 2 Department of Linguistics and Scandinavian Studies, University of Oslo
}

\begin{abstract}
We provide a formal model for the interaction of syntax and pragmatics in the interpretation of anaphoric binding constraints on personal and reflexive pronouns. We assume a dynamic semantics, where type $e$ expressions introduce discourse referents, and contexts are assignments of individuals to discourse referents. We adopt the Partial Compositional Discourse Representation Theory (PCDRT) of Haug (2014b), whereby anaphoric resolution is modelled in terms of a pragmatically-established relation between discourse referents. We integrate PCDRT into the constraint-based grammatical framework of Lexical Functional Grammar (LFG), and show how it is possible to state syntactic constraints on the pragmatic resolution of singular and plural anaphora within this framework.
\end{abstract}

1

\section{INTRODUCTION}

Pronouns are among the most frequently occurring words in many languages, including English, and speakers find no difficulty in using them and, for the most part, determining their reference in a particular context. However, formally analysing the constraints on the interpretation of pronouns in context is a complex matter. In part, this is due to the fact that the interpretation of pronominal reference involves two components of language which are usually considered separate: syntax and pragmatics. While syntax and semantics are widely

Keywords:

anaphora, binding theory, Lexical Functional Grammar, Partial Compositional Discourse Representation Theory 
treated as part of a formal computational system which pairs linguistic form with linguistic meaning, pragmatic interpretation is widely treated as a distinct system which interprets linguistic meaning but which is separate from grammar itself. The interpretation of pronouns is one instance of a linguistic phenomenon which brings into question the sharp separation of pragmatics from the rest of grammar. ${ }^{1}$ The Partial Compositional Discourse Representation Theory (PCDRT) of Haug (2014b) addresses this by modelling anaphoric resolution in terms of a pragmatically-established relation between discourse referents. This creates a clean separation of monotonic from non-monotonic aspects of interpretation, licensing an integration of pragmatics into grammatical modelling without undermining the monotonic nature of the rest of grammar.

In this paper we provide a formal model for the interaction of syntax and pragmatics in the interpretation of anaphoric binding constraints on personal and reflexive pronouns. We implement the model using PCDRT as the semantic framework, but the only information that needs to be available at the syntax-pragmatics interface is a function that takes anaphoric expressions to their antecedents. Our approach is therefore compatible with any theory that models anaphor-antecedent relationships in this way (as opposed to, say, equating anaphor and antecedent variables in the syntax).

Nevertheless, we believe there is considerable value in demonstrating how PCDRT can be integrated into the constraint-based grammatical framework of Lexical Functional Grammar (LFG). The most complex data that we consider in this paper - negative binding constraints on plural pronouns - have to our knowledge not been treated since the work of Berman and Hestvik (1997), which is cast in a framework where Government and Binding-style trees are rewritten as DRSs. This approach to the syntax-semantics interface is hardly in use today, whereas we are assuming a standard, lambda-driven approach to semantic composition. From a formal point of view, LFG and PCDRT naturally complement each other. LFG distinguishes different types of grammatical information and treats them as distinct levels of representation. Their combination produces a model in which syn-

\footnotetext{
${ }^{1}$ For a detailed discussion of other phenomena that point in this direction, see e.g. Chierchia (2004).
} 
tactic, semantic and pragmatic constraints on pronoun resolution can be integrated while remaining distinct. This allows us to extend the empirical coverage of LFG, in particular by providing a complete and formally explicit account of negative binding constraints, which has not been available in previous work.

2

\section{SYNTACTIC BINDING CONDITIONS}

It is clear enough that at least to some extent, the resolution of pronominal anaphora is pragmatically based. Given the following sentence, the hearer cannot determine syntactically or semantically whether he refers to Bertie or to a different individual (available from the discourse or wider context); this determination can only be made pragmatically, based on the context of the utterance.

(1) Bertie knew that he wanted to leave.

Most importantly, the determination of a particular antecedent for a pronoun is not fixed: once it is made, it can be revised if the subsequent discourse provides additional information which contradicts the assignment made. This ability to update the relations between pronouns and antecedents shows that the relation is fundamentally pragmatic. This will be discussed in Section 5 .

At the same time, pronominal binding is generally subject to syntactic constraints of various kinds, defined in terms of a superiority relation between the pronoun and its allowed and disallowed binders, and the syntactic domain in which a pronoun must or must not be bound. The classic binding theory of Chomsky (1981) defines the following constraints:

(2) Binding conditions according to Chomsky (1981, 188):

Principle A: An anaphor (myself, himself, themselves) is bound in its governing category.

Principle B: A pronominal (me, he, him, them) is free in its governing category.

In Chomsky's setting, 'bound' is defined in terms of c-command, and the 'governing category', the domain of binding, is roughly the clause. However, subsequent work has shown that other syntactic domains are also relevant for the definition of binding constraints, and that 
the binding domain can vary for different pronominal elements, even within the same language.

We adopt the binding theory of Dalrymple (1993, 2001), who builds on original work by Bresnan et al. (1985) in proposing four domains which are relevant for anaphoric binding: the Root Domain, i.e. the domain consisting of an entire sentence or utterance; the Minimal Finite Domain, i.e. the minimal syntactic domain containing a finite element; the Minimal Complete Nucleus, i.e. the minimal syntactic domain containing an argument with the grammatical function subject; and the Coargument Domain, i.e. the minimal domain defined by a predicate and the arguments it governs. More recent work on binding theory in LFG adopts and builds on this approach (see in particular Bresnan et al. 2016, Chapters 9 and 10, and references cited there).

The binding domain for each anaphoric element is specified in its lexical entry. For example, the English personal pronouns obey binding constraints defined in terms of the Coargument Domain: a pronoun may not corefer with a superior coargument of the same predicate. In (3b), him may not be interpreted as coreferring either with Alan or Bertie, since all three are arguments of the same predicate, told. ${ }^{2}$ In (3c,d), him may corefer with Alan, since they are arguments of different predicates: for example, in (3c), Alan is the subject of saw, and him is the object of near.
a. $\operatorname{Alan}_{i}$ likes him ${ }_{* i}$.

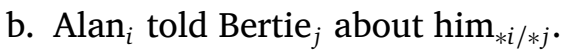
c. $\operatorname{Alan}_{i}$ saw a snake near himi/j.
d. Alan $_{i}$ said that Bertie ${ }_{j}$ likes him $_{i / * j / k}$.

In contrast, the antecedent of the English reflexive pronoun must corefer with a superior element within a different syntactic domain, the Minimal Complete Nucleus. In (4b), the reflexive pronoun rather than the personal pronoun is used to indicate coreference between himself and either Alan or Bertie; in (4c), coreference is allowed between

\footnotetext{
${ }^{2}$ Following standard practice, we use alphabetic subscripts such as $i$ and $j$ to indicate coreference. Later in the paper, we introduce indices as linguistic objects, and we represent the unique index of a phrase by a numeral.
} 
himself and Alan, since both appear in the same Minimal Complete Nucleus; in (4d), coreference is disallowed between Alan and himself, since Alan appears outside the Minimal Complete Nucleus in which himself appears.
a. Alan $_{i}$ likes himself ${ }_{i}$.
b. Alan $_{i}$ told Bertie ${ }_{j}$ about himself ${ }_{i / j}$.
c. Alan $_{i}$ saw a snake near himself .
d. Alan $_{i}$ said that Bertie ${ }_{j}$ likes himself ${ }_{* i / j}$.

These examples illustrate the differing nature of the binding constraints on English personal and reflexive pronouns, encoded in Chomsky's Principles A and B as the difference between bound and free anaphoric elements. Anaphors such as himself obey positive constraints, requiring a particular syntactic relation to hold between anaphor and antecedent (i.e. that the antecedent must be bound by a superior element within the anaphor's binding domain). In contrast, pronominals such as him obey negative constraints, ruling out certain syntactic relations from holding between the pronominal and the superior elements within the relevant domain. As we will see in the following sections, positive constraints on a reflexive pronoun like himself are simpler to state than negative constraints on a personal pronoun like him, particularly when plural reference is brought into the picture.

Superiority

Besides specification of the binding domain, we must also specify the elements within the domain which are relevant for binding constraints: these are the elements which are superior to the anaphoric element within the domain. Superiority is defined in terms of both structural configuration and grammatical prominence.

Structurally, we take functional command to be the relevant configuration. We will return to the exact formalization of this relation in LFG in Section 7, but in theory-neutral terms, an element $x$ functionally commands $y$ iff $x$ and $y$ are coarguments or $y$ is embedded in a coargument of $x$.

A grammatical prominence condition is also relevant: for example, although the subject and object of a transitive predicate functionally command each other, the subject is more grammatically promi- 
nent than the object. Thus, an object reflexive may be bound by a subject coargument, but a subject reflexive may not be bound by an object coargument. For simplicity, we take the relevant prominence condition to be the grammatical function hierarchy (Keenan and Comrie 1977): ${ }^{3}$ a subject binds its coarguments and elements contained in its coarguments, but an object does not bind the subject or elements contained in the subject.

\section{NEGATIVE GONSTRAINTS AND GOREFERENGE}

Although it is certainly true that the negative constraint on a pronoun like him is stateable in terms of the syntactic domain in which it appears, it is vital to note that the constraint against identity of reference with a coargument cannot be enforced simply by constraining the choice of antecedent; for example, by disallowing an anaphor taking a coargument as antecedent. Consider example (5):

(5) Bertie thought that he had seen him.

Here, he and him are coarguments, and he is superior to him; therefore him may not take he as its antecedent. Bertie is not a coargument of either he or him, and so in principle Bertie may serve as antecedent for either pronoun. That is, he may take Bertie as antecedent, and likewise him may take Bertie as antecedent. However, as observed by Wasow (1972), Higginbotham (1983), and Lasnik (1989c), Bertie may not function as antecedent to he and him simultaneously, since this would result in coreference between he and him, and this is not allowed. Note that such a configuration is not ruled out by simple syntactic constraints on where the antecedent of each pronoun can appear. Thus, although the basic constraint is syntactic, its application requires a semantic/pragmatic resolution of reference: the individuals referred to by he and him in (5) may not be the same. Our analysis improves on previous work in LFG in explicitly defining the appropriate notion of coreference, and using this definition in the statement of negative constraints.

\footnotetext{
${ }^{3}$ See Dalrymple (1993, Chapter 5) and Bresnan et al. (2016, 218, 246-247, 276) for discussion of additional conditions that have been shown to be relevant to defining grammatical prominence, including the role of linear precedence relations and the thematic hierarchy.
} 
Plural reflexives are subject to the same positive constraint as singular reflexives: a plural reflexive must corefer with a superior antecedent within the Minimal Complete Nucleus, and long-distance or split antecedents are not acceptable.

$$
\begin{aligned}
& \text { a. } \left.\left[\operatorname{Alan}_{i} \text { and Bertie }\right]_{j}\right]_{i+j} \text { like themselves } \\
& \text { b. } \left.{ }^{*}\left[\operatorname{Alan}_{i} \text { and Bertie }\right]_{j}\right]_{i+j} \text { said that Charlie }{ }_{k} \text { likes } \\
& \text { themselves }_{i+j} \text {. } \\
& \text { c. }{ }^{*} \text { Alan }_{i} \text { confronted Bertie }_{j} \text { with themselves }{ }_{i+j} \text {. }
\end{aligned}
$$

In (6), the proper names Alan and Bertie each bear an index, and the coordinated phrase Alan and Bertie also bears a separate, complex index constructed from the conjuncts, as we discuss in Section 9.1. The reflexive must have the same (simple or complex) index as its antecedent.

The situation with plural personal pronouns is considerably more complex. Like singular personal pronouns, plural personal pronouns obey a negative constraint: in (7), them may not have the same index as its coargument Alan and Bertie, just as in example (3).

(7) $\left.*\left[\operatorname{Alan}_{i} \text { and Bertie }\right]_{j+j}\right]_{i \text { like them }}{ }_{i+j}$.

A nonoverlapping (disjoint) relation between the pronoun and its coarguments is uncontroversially acceptable, similar to the requirement for singular pronouns to be noncoreferent with coarguments, as shown in (8).

(8) $\left[\text { Alan }_{i} \text { and Bertie }\right]_{j+j}$ like them ${ }_{k+l}$.

However, with plural pronouns and plural coarguments, other patterns are possible:

A. The index of the coargument is properly included in the index of the pronoun:

[Alan $_{i}\left(\right.$ and Bertie $\left.\left.e_{j}\right)\right]_{i / i+j}$ like(s) them th $_{i+j+k}$.

B. The index of the pronoun is properly included in the index of its coargument:

$\left[\text { Alan }_{i}, \text { Bertie }_{j} \text {, and Charlie }\right]_{i+j+k}$ like him $_{i} /$ them $_{i+j}$.

C. The index of the pronoun overlaps with the index of the coargument, but without an inclusion relation:

$\left.\left[\text { Alan }_{i} \text { and Bertie }\right]_{j+j}\right]_{i k e}$ them $_{j+k}$. 
A fourth pattern has been claimed to be relevant in some of the literature on pronominal binding:

D. The index of the pronoun is the sum of the indices of the coarguments, but not identical to any coargument:

Alan $_{i}$ told Bertie about them $_{i+j}$.

The grammatical status of these patterns is controversial, and various positions have been taken in the literature as to their acceptability, as we now outline. To avoid confusion due to the varying judgements that have been reported, we explicitly mark each example with the judgement reported in the cited work.

4.1 Pattern A: Index of coargument is properly included in index of pronoun

Some simple examples conforming to this pattern have been judged as ungrammatical:
a. ${ }^{*} \mathrm{He}_{i}$ represented them ${ }_{i+}$ (Seeley 1993, 309)
b. "Bill ${ }_{i}$ represented them ${ }_{i+}$. (Seeley 1993, 309)
c. ${ }^{*} \mathrm{John}_{i}$ told them ${ }_{i+j}$ that Mary ${ }_{j}$ should leave. (Lasnik 1989a, 151)

However, many other examples conforming to this pattern have been judged as acceptable:

(10) a. $\checkmark \mathrm{He}_{i}$ talked about them ${ }_{i+}$ (Fiengo and May 1994, 43)

b. $\checkmark$ Bill $_{i}$ was quite pleased [that Mary defended them $_{i+j}$ ]. (Seeley 1993, 308)

c. $\checkmark$ Bill $_{i}$ was happy [because Mary had protected them $_{i+j}$ ]. (Seeley 1993, 308)

d. $\checkmark$ Sam $_{i}$ is telling Tom $_{j}$ not to praise them ${ }_{i+j}$. (Seeley 1993, 308)

e. $\checkmark$ Bill $_{i}$ was surprised that $\left[\mathrm{Mary}_{j}\right.$ 's representing them $_{i+j}$ at the trial] had caused such problems. (Seeley 1993, 308)

f. $\checkmark \mathrm{John}_{i}$ wants Mary $_{j}$ to represent them $\mathrm{i}_{i+j}$. (Berman and Hestvik 1997, 5)

g. $\checkmark\left[\mathrm{John}_{i}\right.$ 's mother $_{j}$ protected them ${ }_{i+j}$ from the robbers. (Berman and Hestvik 1997, 6)

h. $\checkmark\left[\text { The woman who loved } \mathrm{John}_{i}\right]_{j}$ represented them ${ }_{i+j}$ at the trial. (Berman and Hestvik 1997, 6) 
i. $\checkmark\left[\text { Bill }_{i} \text { and Mary }\right]_{j+j}$ were asked to appear before the committee. But Bill ${ }_{i}$ fell ill and had to be excused. $\mathrm{John}_{k}$ said that Mary represented them $_{i+j}$. (Berman and Hestvik 1997, 7)

It has been claimed that increasing the number of coarguments whose reference is included in the reference of the pronoun degrades acceptability; Seeley (1993) provides the following judgements:

(11) a. ?The doctor ${ }_{i}$ told the patient ${ }_{j}$ [that the nurse ${ }_{k}$ would protect them ${ }_{i+j+k}$ during the storm]. (Seeley 1993, 313)

b. ??The doctor ${ }_{i}$ said [that the patient ${ }_{j}$ told the nurse ${ }_{k}$ about them $_{i+j+k}$ ]. (Seeley 1993, 313)

Nevertheless, the large number of acceptable examples of Pattern A indicate that syntactic constraints do not rule out this pattern, but that unacceptable examples are ruled out by some combination of semantic or pragmatic conditions.

Several instances of this pattern reported in the literature involve first-person singular subjects with a coargument first-person plural personal pronoun:

(12) a. $\checkmark$ I expect us to meet John at the party. (Fiengo and May 1994, 44)

b. $\checkmark$ I believe us to have been cheated. (Kiparsky 2002, 20)

c. $\checkmark$ I prefer to call us rape statistics. (Kiparsky 2002, 20)

d. $\checkmark$ I want us to be friends. (Kiparsky 2002, 21)

e. $\checkmark$ We have a terrific team. I really like us. (Kiparsky 2002, 19)

For reasons that are not clear to us, reported judgements of the reverse pattern (We...me) are more often judged as unacceptable, as we discuss below.

4.2 Pattern B: Index of pronoun is properly included in index of coargument

Some examples of Pattern B have also been judged as ungrammatical:

a. *They ${ }_{i+}$ like him $_{i}$. (Lasnik 1989b, 125; Seeley 1993, 309)

b. $*\left[\mathrm{John}_{i} \text { and Mary }\right]_{i+j}$ are taking care of him ${ }_{i}$. (Kiparsky 2002, 20) 
However, just as with Pattern A, many examples of this pattern have been judged as acceptable:

(14) a. $\checkmark\left[\text { Richard }_{i} \text { and } \text { Pat }_{j}\right]_{i+j}$ both regard $\operatorname{him}_{i} /$ her $_{j}$ as innocent. (Kiparsky 2002, 20)

b. $\checkmark\left[\mathrm{John}_{i} \text { and } \text { Mary }_{j}\right]_{i+j}$ talked about him ${ }_{i}$. (Fiengo and May 1994, 43)

c. $\checkmark\left[\mathrm{John}_{i} \text { and } \mathrm{Mary}_{j}\right]_{i+j}$ often connive behind their colleagues' backs to advance the position of one or the other. This time, they $y_{i+j}$ managed to get her $_{j}$ a position in the front office. (Berman and Hestvik 1997, 8)

d. $\checkmark$ [The men $_{i}$ and Mary $]_{i+j}$ talked about them ${ }_{i}$. (Fiengo and May 1994, 43)

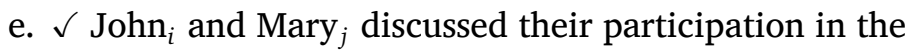
upcoming contest with Bill $_{k}$. They Th+j+k $_{i+1}$ expect them ${ }_{i+j}$ to win. (Berman and Hestvik 1997, 8)

f. Acceptable "for many speakers": $\left[\text { Felix }_{i} \text { and } \text { Lucie }_{j}\right]_{i+j}$ authorized her $_{j}$ to be their representative. (Reinhart and Reuland 1993, 677)

Distributivity has been claimed to be a factor in the acceptability of Pattern B examples. Reinhart and Reuland (1993) claim that there is a contrast between the examples in (15), where both forces a distributive reading:

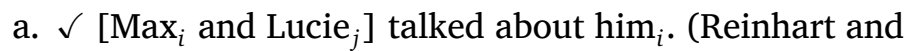
Reuland 1993, 677)

b. * $\left[\right.$ Both Max $_{i}$ and Lucie $\left.{ }_{j}\right]$ talked about him ${ }_{i}$ (Reinhart and Reuland 1993, 677)

According to Reinhart and Reuland (1993) and Kiparsky (2002), Pattern B sentences are ungrammatical only under a distributive reading, but are fine under a collective reading. Strikingly, however, Seeley (1993) judges the examples with both in (16b,c) as grammatical, in contrast to (16a), which he judges as ungrammatical. Seeley observes that the presence of both plays a 'key role'.

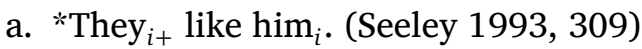
b. $\checkmark\left[\text { Bill }_{i} \text { and } \text { Mary }_{j}\right]_{i+j}$ both introduced him ${ }_{i}$. (Seeley 1993, 308)
c. $\checkmark$ They $_{i+}$ both introduced him ${ }_{i}$ (Seeley 1993, 308) 
Again, then, we take the large number of acceptable Pattern B examples, together with the lack of agreement about distributivity, as demonstrating that Pattern B examples do not violate syntactic constraints, but should be accounted for in semantic or pragmatic terms.

As noted above, first-person examples seem to differ to some degree between Pattern A and Pattern B, in that more Pattern B examples than Pattern A examples are judged as ungrammatical.
a. "We like me. (Lasnik 1989b, 125)
b. "We watched me leaving (in the mirror). (Chomsky 1973, cited in Lasnik 1989d)

Fiengo and May (1994) judge (18b) as 'worse' than (18a), its Pattern A counterpart:
a. $\checkmark$ I expect us to meet John at the party. (Fiengo and May 1994, 44)
b. 'worse': We expect me to meet John at the party. (Fiengo and May 1994, 44)

Nevertheless, some Pattern B first-person examples are judged as acceptable:

(19) a. $\checkmark$ We made John president and me vice-president. (Fiengo and May 1994, 44)

b. $\checkmark$ By an overwhelming majority, we preferred me. (Kiparsky 2002, 19)

\subsection{Pattern C: Index of pronoun overlaps with index of coargument without inclusion}

Although much attention has been paid to cases A and B where the index of the pronoun properly includes the index of a coargument or vice versa, no one to our knowledge has discussed the third logically possible pattern of overlapping indices, where neither index properly includes the other. We therefore find no such examples in the literature, but we can easily construct them with appropriate changes to the examples in sections A and B:

(20) a. Bill $l_{i}$ was happy [because $\left[\text { Mary }_{j} \text { and her } \operatorname{dog}_{k}\right]_{j+k}$ had protected them ${ }_{i+j}$ ]. $(\approx 10 \mathrm{c})$ 
b. $\left[\text { Richard }_{i} \text { and Pat }\right]_{i+j}$ both regard $\left[\text { him }_{i} \text { and his wife }\right]_{i+k}$ as innocent. $(\approx 14 \mathrm{a})$

We judge these examples acceptable. And to the extent that the original examples (10c) and (14a) have been judged acceptable, there is no reason to expect a different judgement in this case.

\subsection{Pattern D: Index of pronoun is sum of indices of coarguments}

This pattern is rarely discussed, though it is a focus of attention in work by Seeley (1993) and Berman and Hestvik (1997). Note that Pattern D is only possible with predicates that can take more than two arguments, since the pronoun must fill one argument slot and refer to a group composed of individuals that fill at least two of the other argument slots. This more or less excludes one common class of ditransitives, the transfer verbs, as a source of examples, because the object is typically inanimate and the recipient/goal typically animate. Fiengo and May (1994) provide the example in (21a) in support of their claim that Pattern D is acceptable, while Seeley provides the example in (21b) in support of the opposite claim. Berman and Hestvik (1997) discuss both examples and agree with both judgements, and also provide the example in (21c). They furthermore claim - without examples - that similar ungrammatical binding patterns can be constructed with the verbs assign to, deny to, cede to, compare to, consign to, entrust to, explain to, leave to, offer to, point out to, promise to, and reveal to. Finally, Kiparsky (2002) provides example (21d) with the judgement as indicated.

(21) a. $\checkmark \mathrm{John}_{i}$ talked to Mary ${ }_{j}$ about them ${ }_{i+j}$. (Fiengo and May 1994, 40; Berman and Hestvik 1997, 24)

b. *Bill ${ }_{i}$ told Mary $_{j}$ about them ${ }_{i+j}$. (Seeley 1993, 307; Berman and Hestvik 1997, 6)

c. (At their wedding reception, John and Mary were speaking to Bill and Sue.) * $\mathrm{John}_{i}$ said that he ${ }_{i}$ wanted $\left[\mathrm{PRO}_{i}\right.$ to photograph Mary ${ }_{j}$ for them ${ }_{i+j}$. (Berman and Hestvik 1997, 25)

d. ${ }^{*} \mathrm{John}_{i}$ confronted Bill $_{j}$ with them ${ }_{i+j}$. (Kiparsky 2002, 21)

In these examples, them does not corefer with either of its coarguments, but overlaps in reference with both such that its reference is 
exhausted by its coarguments. Seeley (1993) and Berman and Hestvik (1997, 20-21, 24-27) claim that this is disallowed, and that it is necessary not only to prohibit coreference between the discourse referents introduced by a pronoun and a (superior) coargument, but also between the pronoun's discourse referent and the sum of its (superior) coarguments. Berman and Hestvik also discuss (21a) and judge it as acceptable, claiming that its acceptability is due to special properties of talk that remain unclear. ${ }^{4}$

Nevertheless, the evidence seems inconclusive to us. Berman and Hestvik (1997, Section 5) admit that the empirical status of these examples is somewhat unclear. For what it is worth, note that it is possible to find naturally occurring examples with first and second person arguments as in (22).

(22) Khushi looks up at Arnav with tear-filled eyes: Would you tell me about us? How did we meet? When did we fell in love?

Everything from the beginning?

(http://fast-forward-by-tia.blogspot.no/)

The theory that we develop below predicts that Pattern D is grammatical, although it is possible to rule it out in our model; we return to this issue in Section 9.5.

In sum, previous work on binding involving plural pronouns or antecedents is unanimous in ruling out strict coreference between a plural pronoun and a superior coargument, but there is a great deal of variation in judgements on cases of overlap or inclusion (for additional discussion of this point, see Büring 2005, Chapter 9). It is well known that binding possibilities are influenced by lexical, structural, and contextual factors that are not yet completely understood; see Jackendoff (1992), Reinhart and Reuland (1993), Berman and Hestvik (1997), and Park (2012) for discussion. We take the position that syntactic binding constraints for English singular and plural personal pronouns rule out coreference between the pronoun and its superior coarguments, but that overlap or inclusion is permitted. This means that the unacceptable examples in this section that do not involve strict coref-

\footnotetext{
${ }^{4}$ They do, however, rule out an analysis according to which Mary does not c-command out of the PP in (21a) because they judge John talked to Mary ${ }_{i}$ about herself $_{i}$ acceptable.
} 
erence with a coargument are not ruled out by syntactic constraints, but are unacceptable for other reasons.

The most successful attempts to deal with anaphoric resolution, especially across sentences, have been developed within the tradition of dynamic semantics. We follow that tradition here, in particular the version developed by Kamp and Reyle (1993) and Kamp et al. (2011), Discourse Representation Theory (DRT). ${ }^{5}$ In dynamic semantics, the meaning of a sentence is not its truth conditions, but its context change potential, made precise as a relation between assignments of individuals to discourse referents at different points in the discourse. Consider the DRS in (23).

(23) A linguist arrived.

\begin{tabular}{|l|}
\hline$x$ \\
\hline $\begin{array}{l}\text { linguist }(x) \\
\text { arrive }(x)\end{array}$ \\
\hline
\end{tabular}

(23) is interpreted as a relation between an 'input' assignment $i$ and an 'output' assignment $o$ such that $o$ is like $i$ except it assigns some individual to $x$ that is in the denotation of linguist and arrive. ${ }^{6}$ This is shown in (24), where $\mathfrak{I}$ is the interpretation function assigning relational meanings to predicate constants and $i \subset_{\{x\}} o$ means that $o$ is like $i$ except in assigning some value to $x$.

(24) Interpretation of (23) as a relation between input and output assignments (Kamp and Reyle 1993):

$$
\left\{\langle i, o\rangle \mid i \subset_{\{x\}} \circ \wedge o(x) \in \Im(\text { linguist }) \wedge o(x) \in \Im(\text { arrive })\right\}
$$

Although in this setting the meaning of a sentence is a relation between assignments, there is a natural way to get to truth conditions,

\footnotetext{
${ }^{5}$ Similar ideas are found in Heim (1982) and many later versions of dynamic semantics.

${ }^{6}$ We use $i$ and $o$ as variables over states when these function as input and output states of a DRS, but $s$ when we talk about states more generally.
} 
by taking $i$ to be the empty assignment and requiring the existence of some assignment $o$. That is, we define truth (using $s_{\emptyset}$ for the empty assignment) so that (23) is true iff there is an o such that $\left\langle s_{\emptyset}, o\right\rangle$ is in (24). It is easily seen that this yields the same truth conditions as for the first-order translation of (23), which is $\exists x$.linguist $(x) \wedge$ arrive $(x)$.

Nevertheless, although the truth conditions turn out the same as in first-order logic, there is a difference in the predictions about anaphoric accessibility. The idea is to use $o$, the 'output' assignment of this sentence, as the input assignment for the subsequent discourse, thereby making $x$ accessible for anaphoric uptake.

(25) He sat down.

\begin{tabular}{|l|}
\hline$y$ \\
\hline sit.down $(y)$ \\
$y=?$
\end{tabular}

Here the anaphor is associated with a condition $y=$ ?, which we can interpret as an instruction to find an antecedent. If this sentence follows sentence (23), $y$ can be equated with $x$, with the result that the two-sentence discourse means that there is some individual who is a linguist, arrived, and sat down.

However, this treatment of anaphora means that the DRS in (25) has no interpretation at all until such an antecedent is found. This makes the framework representational: the DRSs are essential ingredients of the analysis and cannot be 'translated away' the way lambda terms can be in the Montagovian tradition. Moreover, because (25) has no meaning until the antecedent is found, we cannot make sense of the intuition that, in many cases, the sentence containing the anaphor will constrain the resolution. For example, it is likely that it in It meowed will be resolved to some animal making the appropriate sound, but we cannot model this if It meowed does not have a meaning until a referent is found.

This representational nature of Kamp and Reyle's DRSs was an obstacle to compositionality. In response, Muskens (1996) developed a compositional version of DRT, CDRT. The leading idea is to inject assignments into the object language, with explicit quantification over information states, plus an 'interpretation function' $v$ which assigns an 
individual inhabitant to each discourse referent in every state. That is, discourse referents (or registers, as they are often called in CDRT) are no longer simply variables over individuals but are reified as terms of a separate type, $\pi$, which are 'interpreted' by the function $v$. Furthermore, we have axioms which guarantee that $v$ actually works as an assignment.

With that in place, we can view DRSs such as (23) as abbreviations for more complex lambda terms. Instead of interpreting the DRS as a relation between assignments in the metalanguage, we now expand it as $\lambda i . \lambda$ o.P in the object language, where $P$ is the contents of the DRS. As before, those contents have two parts: a universe and a set of conditions. In the conditions, we expand a discourse referent $x$ as $v(o)(x)$. Observe that $x$ here is a constant (of type $\pi$ ), but when we plug it into the $v$ function, we get a term of type $e$, the inhabitant of that discourse referent. So in practical terms $x$ works like a variable. We interpret the declaration of discourse referents in the universe of a DRS as a constraint that the input and output states of that DRS, $i$ and $o$, differ at most with respect to the values of those variables, i.e. (for (23)), $\forall \delta . \delta \neq x \rightarrow v(i)(\delta)=v(o)(\delta)$.

In sum, we now have (26) as the expansion of (23).

(26) Content of (23) in CDRT (Muskens 1996):

$\lambda i . \lambda o . \forall \delta . \delta \neq x \rightarrow v(i)(\delta)=v(o)(\delta) \wedge \operatorname{linguist}(v(o)(x)) \wedge \operatorname{arrive}(v(o)(x))$

Compared to (23)-(24), what has happened here is that the assignments, which only played a role in the metalanguage interpretation (24) in the DRT approach, are now part and parcel of the object language (26). Nevertheless, we can get to the truth conditions in a very similar way by saturating $i$ with the empty assignment $s_{\emptyset}$ and existentially closing $o$.

What about the unresolved anaphor in (25)? It is not trivial to give a model-theoretic semantics for a condition like $y=$ ?, which seems irreducibly procedural: first we pick an antecedent and then we interpret the whole thing semantically. Muskens' solution was to simply use coindexation, which in CDRT terms means that we use the same discourse referent for both he and a linguist. But this means the syntax has to take care of anaphoric resolution, which is problematic for several reasons, as noted by Beaver (2002). 
We therefore follow Haug (2014b), who partialized the underlying logic to allow for model-theoretic representation of unresolved anaphora in his Partial CDRT (PCDRT). In a given state, $v$ now acts as a partial assignment, which means that we can identify unused discourse referents. Instead of using a constant $x$, CDRT uses a function expression picking out the first unused discourse referent in the input state ( $i)$. We forego details here, but the reader should bear in mind that $x$ in (26) is not in fact a free variable (or a constant), but a discourse referent functionally dependent on $i$.

More importantly, anaphoric discourse referents are translated as any other, without any coindexation. They are, however, marked as anaphoric; we represent anaphoric discourse referents with an overbar $(\bar{x})$. The truth definition then requires all anaphoric discourse referents to corefer with an accessible antecedent, as in (27): otherwise there is a truth value gap. This latter effect is achieved by Beaver's unary presupposition connective $\partial$ (Beaver 1992), which maps $\partial(\phi)$ to true if $\phi$ is true and to the undefined truth value otherwise.

(27) Condition on antecedency for anaphoric discourse referents:

$$
\partial\left(v(s)(\bar{x})=v(s)\left(\mathscr{A}_{s}(\bar{x})\right)\right)
$$

This condition requires $\bar{x}$ to be identical to its antecedent $\mathscr{A}_{S}(\bar{x})$ in the state $s$, as specified by the antecedency function $\mathscr{A}$, thus yielding coreference or, if $\mathscr{A}_{s}(\bar{x})$ is itself bound by an operator, covariation. Notice that $\bar{x}$ and its antecedent must both be defined in the same state $s$; this yields the usual operator-induced restrictions on anaphoric accessibility, as in DRT. We often omit the subscript $s$ on the anaphoric relation $\mathscr{A}$, while retaining the requirement that the anaphoric relation $\mathscr{A}$ is defined only between discourse referents in the same state. ${ }^{7}$

$\mathscr{A}$ is a function from discourse referents in a particular state $s$ to discourse referents (in the same state $s$ ). It is a composite function:

(28) Definition of $\mathscr{A}$ in a state $s:{ }^{8}$

$$
\mathscr{A}_{s}(x) \equiv \mathscr{I}_{s}^{-1}\left(\mathscr{R}\left(\mathscr{I}_{s}(x)\right)\right)
$$

${ }^{7}$ Haug (2014b) in addition assumes a constraint $\mathscr{A}_{s}(x)<x$ requiring the antecedent to precede the pronoun, but the (marked) possibility of cataphoric resolution shows that this constraint is non-monotonic.

${ }^{8}$ Haug (2014b, 497, ex. 69) defines $\mathscr{A}$ in terms of $\mathscr{R}^{*}$, the transitive closure of $\mathscr{R}$. We define and discuss $\mathscr{R}^{*}$ in Section 7. 
The function $\mathscr{I}_{s}$ maps the discourse referents in a state $s^{9}$ to objects which we will call 'indices', which introduce discourse referents; crucially, as we will see, indices are accessible to syntactic representations and constraints. $\mathscr{I}^{-1}$ is the inverse mapping, a function from indices back to the discourse referents they introduce (in a particular state $s$ ). The core of pragmatic anaphora resolution is then the function $\mathscr{R}$, which maps indices to antecedent indices. This allows us to keep the simple idea underlying the coindexation approach, namely that anaphoric relations are just relations between syntactic tokens, but without presupposing that the resolution is actually done in the syntax. ${ }^{10}$ We thus have the following set-up: indices, which are syntactically accessible, introduce discourse referents; by mapping from discourse referents to indices, then from indices to antecedent indices, and finally from antecedent indices to discourse referents, we obtain a mapping between discourse referents and their antecedent discourse referents (in a particular state).

(29) The relations $\mathscr{A}, \mathscr{R}$ and $\mathscr{I}$ :

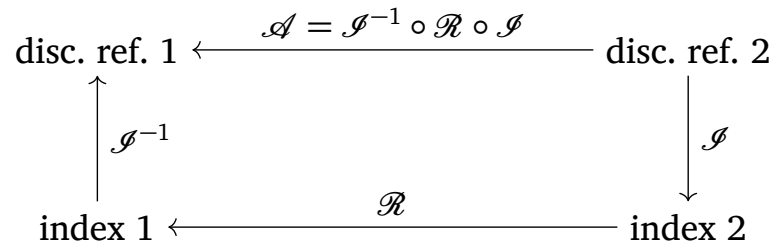

Since $\mathscr{A}$ is uniquely determined by $\mathscr{R}$, we will use constraints on $\mathscr{R}$ to capture the constraints of binding theory. But first, in the following section, we integrate the model with the framework of LFG.

In this section we show how the PCDRT approach to anaphora can be integrated with the grammatical framework of Lexical Functional Grammar (LFG), to provide a formal model of the interaction between

\footnotetext{
${ }^{9}$ As with $\mathscr{A}$ we will often omit the subscript $s$ on $\mathscr{I}$.

${ }^{10}$ There are also technical advantages over the view that anaphoric relations hold between discourse referents in context, because the semantics quantifies over contexts in a way that would scope over anaphoric resolutions, whereas anaphoric resolution between indices/syntactic tokens will always be scopeless. See Nouwen $(2003,140)$ and Haug (2014b, 482-483) for details.
} 
syntax and pragmatics. LFG is a constraint-based, non-derivational framework for grammatical analysis, developed by Kaplan and Bresnan (1982), and presented in detail by e.g. Dalrymple (2001), Falk (2001) and Bresnan et al. (2016). A crucial element of the LFG framework is that different types of grammatical information are distinguished from one another and treated as distinct levels of grammatical representation, related by means of piecewise functions called projections. LFG therefore provides an ideal grammatical framework into which to integrate PCDRT, with its clear representational separation of semantics and pragmatics.

For example, the phrasal structure of a clause, the $c$ (onstituent)structure, is treated as one level of grammatical representation, represented by means of a phrase-structure tree. In contrast, functional syntactic relations, e.g. grammatical functions such as subject and object, are treated at a separate level, $f$ (unctional)-structure, represented as an attribute-value matrix. So, for the English sentence in (30), the surface phrasal structure, the c-structure, can be represented as in (31), and the abstract syntactic structure, the f-structure, can be represented as in (32). Following standard LFG conventions, we represent only those features of f-structure that are relevant for the discussion at hand, omitting features encoding information about person, number, gender, tense, aspect, and other grammatical information.

(30) Henry laughed.

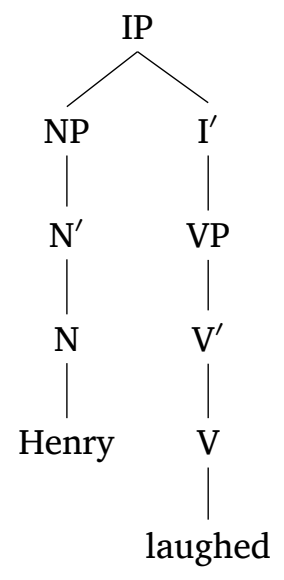




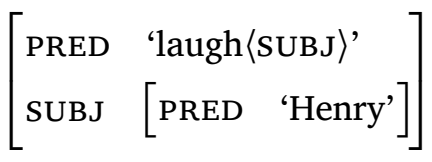

These two grammatical modules are related via a projection function $\phi$, which maps c-structure nodes to their corresponding f-structures. Constraints on the $\phi$ function are stated in terms of functional descriptions associated with nodes of the phrase structure tree; these functional descriptions use the variable $*$ to represent the c-structure node on which the constraint appears, and the variable $\hat{*}$ to represent the mother of the node bearing the constraint. The f-structure projected from a c-structure node is therefore obtained by applying the function $\phi$ to $*$, i.e. $\phi(*)$, and the f-structure projected from a c-structure node's mother is obtained by applying $\phi$ to $\hat{*}$, i.e. $\phi(\hat{*})$. These functions are usually abbreviated by the f-structure metavariables $\downarrow$ and $\uparrow$ :

$$
\begin{aligned}
& \text { a. } \downarrow \equiv \phi(*) \\
& \text { b. } \uparrow \equiv \phi(\hat{*})
\end{aligned}
$$

These metavariables enable concise statements of the constraints on the relation between c-structures and f-structures. For example, in English the specifier of IP is associated with the grammatical role of subject. We represent this by means of the following phrase-structure rule:

$$
\text { IP } \rightarrow \underset{(\uparrow \text { SUBJ })=\downarrow \quad}{\mathrm{NP}} \quad \begin{gathered}
\mathrm{I}^{\prime} \\
\end{gathered}
$$

The annotation ( $\uparrow$ SUBJ) $=\downarrow$ on the constituent in the specifier of IP requires that the f-structure projected from the NP $(\downarrow)$ supply the value of the attribute SUBJ in the f-structure projected from the NP's mother ( $\uparrow)$. The annotation $\uparrow=\downarrow$ on the I' requires the f-structure projected from the $I^{\prime}(\downarrow)$ and the f-structure projected from the IP ( $\left.\uparrow\right)$ to be the same. Ex. (35) shows the c-structure for (30), just as in (31) but with annotated constraints referring to the f-structure on each node. The f-structure is as in (32), and the projection function $\phi$ is represented by means of arrows between the two structures. 
(35)

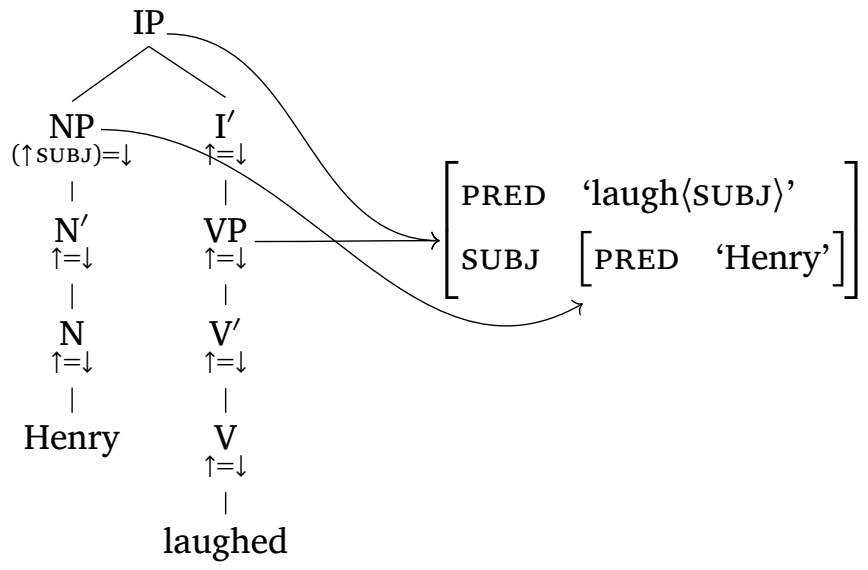

The concept of projection functions between different levels of grammatical representation was generalised by Kaplan (1989) in terms of a 'projection architecture' modelling the different levels of linguistic structure and the relations among them. The full inventory of levels of grammatical representation and the projection functions relating them are a matter of debate, but the details do not concern us here. ${ }^{11}$

Of crucial importance for the present topic, however, is the interface between syntax and semantics. Work on semantics in LFG makes use of the 'glue' theory of the syntax-semantics interface (Dalrymple 2001; Asudeh 2012), according to which meanings are paired with logical expressions which constrain their composition. In standard approaches to glue semantics within LFG, meanings are paired with logical formulae over $s$ (emantic)-structures, projected from f-structures via the projection function $\sigma$. For example, the meaning of the proper name, Henry, is paired with a semantic structure projected from the SUBJ f-structure. For ease of exposition, we introduce labels such as $l$ and $h$ to facilitate reference to different parts of the f-structure. As is standard, we use a subscript $\sigma$ to refer to the s-structure projected from a given f-structure. Thus, $h_{\sigma}$ is the semantic structure corresponding to the f-structure labeled $h$.

\footnotetext{
${ }^{11}$ On the projection architecture of LFG, see e.g. Bögel et al. (2009), Dalrymple and Mycock (2011), Dalrymple and Nikolaeva (2011), Giorgolo and Asudeh (2011), Asudeh (2012, 53), and Mycock and Lowe (2013).
} 


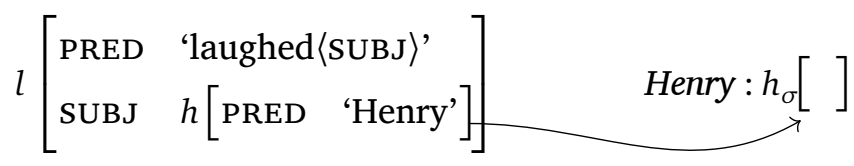

S-structure is an interface structure for modelling the influence of syntax on semantic compositionality. Recent work on semantic structure has emphasised its internal complexity, particularly in regard to the embedding of s-structures within other s-structures (Asudeh and Giorgolo 2012; Asudeh et al. 2014) and the types of features that are present within s-structures (Dalrymple and Nikolaeva 2006).

We propose that the indices between which anaphoric relations hold in PCDRT are a component of semantic structure. It has been observed in the glue literature (e.g. Kokkonidis 2008, 63) that the empty 'placeholder' semantic structures typically used in (higher-order) glue semantics would - under the standard, set-theoretic interpretation of LFG attribute-value structures - in fact lead to an unwanted lack of differentiation among semantic structures. To guarantee that we can keep semantic structures apart, it is necessary to equip them with a uniquely identifying element working in much the same way as the semantic form value of the PRED feature at f-structure (Kaplan and Bresnan 1982, 225). We take these uniquely identifying elements to be the indices discussed in the previous section, and we assign indices as the values of the s-structure feature INDEX.

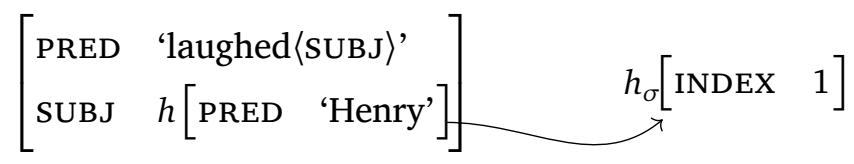

In (37), then, the index 1 uniquely identifies the semantic structure $h_{\sigma}$. We assume that all semantic structures that introduce discourse referents are associated with an index (though, as we will see, not all indices are associated with semantic structures: complex indices can also be constructed by combining contextually salient indices). Indices might also be associated with semantic structures introducing discourse referents over events or times, but for the purposes of binding theory we are only interested in type $e$ (nominal) discourse referents.

Thus, the value of ( $h_{\sigma}$ INDEX) is a unique index that is mapped to some discourse referent in a given information state $s$ by the func- 
tion $\mathscr{I}_{s}^{-1}$ discussed above. In complex contexts involving embedded DRSs and hence several information states, there will be several functions $\mathscr{I}_{s}^{-1}$ potentially mapping different semantic indices to the same discourse referent (interpreted in different states): see Haug (2014b) for more details on how this works. For our purposes, however, we do not need to deal with embedded DRSs or different information states, and we can therefore make the simplifying assumption that semantic indices map one-to-one to discourse referents. To ease the exposition we can use integers $n$ for the values of INDEX attributes, and $x_{n}$ for the corresponding discourse referents.

In the next sections we show how syntactic constraints on the interpretation of pronouns can be defined in terms of an $\mathscr{R}$ relation between indices.

7

REFLEXIVE PRONOUNS

AND POSITIVE GONSTRAINTS

We begin with a relatively simple example of the positive binding constraint on English reflexive pronouns, before moving on to consider the more complex issue of negative constraints. In this section, it will largely be sufficient to adapt existing machinery and analyses to our setting. This will introduce standard aspects of LFG's binding theory, which we then extend to deal with negative constraints.

The positive binding constraint on English reflexives is stated in (38):

(38) English reflexives must have a superior antecedent within the

Minimal Complete Nucleus binding domain.

The Minimal Complete Nucleus is the minimal f-structure containing a SUBJ function. This means that in the following example, the reflexive pronoun himself may in principle corefer with either the subject Alan or the object Bertie.

(39) Alan $_{i}$ told Bertie about himself $_{i / j}$.

Let us assume that in context the most natural interpretation is where himself is coreferent with the subject, Alan. As discussed in the previous section, the indices of words introducing discourse referents appear as the value of the feature INDEX in the s-structure of the word concerned. In this case, then, we have the following $\mathscr{R}$ relation: 
(40) The $\mathscr{R}$ relation: $\operatorname{Alan}_{i}$ told Bertie about himself ${ }_{i}$

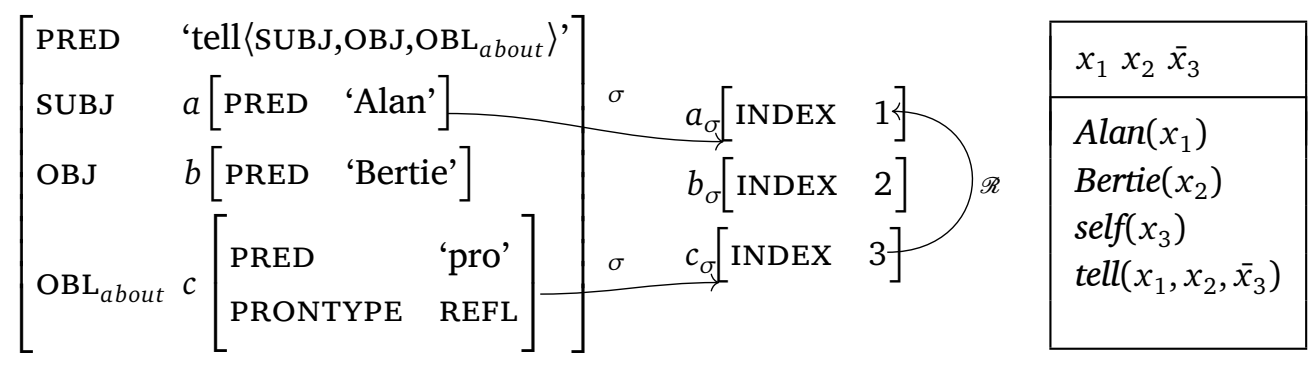

The index 1 maps to the discourse referent $x_{1}$ by the inverse function $\mathscr{I}_{s}^{-1}, 2$ maps to $x_{2}$, and 3 maps to $x_{3}$. In the context under consideration, the index 1 of the subject Alan is assigned as the antecedent of the index 3 belonging to the reflexive pronoun; this is modelled by means of the function $\mathscr{R}$. In another context, the resolution $\mathscr{R}(3)=2$ (the reflexive is bound by the object) could have been more likely. However, the grammar rules out the possibility that the reflexive has e.g. a sentence-external antecedent, with $\mathscr{R}(3)$ resolved to an index other than 1 or 2 . So we want to recast (38) as a constraint on the resolution of $\mathscr{R}$.

In order to state (38) as a constraint on $\mathscr{R}$ in the LFG formalism, we need to express the notions of superiority and binding domain. The latter concept is relatively straightforward as it can be expressed by a formula of the general form shown in (41): ${ }^{12}$

(41) General relation between an anaphor with f-structure $\uparrow$ and its binders:

$$
\left(\left(\mathrm{GF}^{+} \uparrow\right) \mathrm{GF}_{\mathrm{ant}}\right)
$$

This expression represents the set of potential f-structure antecedents of the reflexive pronoun himself. In this expression, $\uparrow$ is the f-structure corresponding to the preterminal node dominating the word himself; $\mathrm{GF}$ is a variable over grammatical functions (SUBJ, OBJ, etc.); $\mathrm{GF}^{+}$is a sequence of grammatical functions $\mathrm{GF}$, a path through the f-structure ending in $\uparrow$; and $\mathrm{GF}_{\text {ant }}$ is the grammatical function of the antecedent. The expression $\left(\mathrm{GF}^{+} \uparrow\right)$ refers to any f-structure properly containing

${ }^{12}$ For a full explication, see Dalrymple (1993, 2001) and Bresnan et al. (2016). 
the f-structure $\uparrow$, and the antecedent of the reflexive bears the grammatical function $\mathrm{GF}_{\text {ant }}$ within the f-structure $\left(\mathrm{GF}^{+} \uparrow\right)$.

(42) Schematic syntactic relation between the anaphor and its antecedent:

$f\left[\begin{array}{ll}\mathrm{GF}_{\text {ant }} & {[\text { ANTECEDENT }]} \\ \mathrm{GF} & \cdots[\text { [REFLEXIVE }(\uparrow \text { in }(41))]\end{array}\right]$

Notice that the form of this constraint makes sure that the antecedent functionally commands the reflexive as defined in Section 2.2: the antecedent bears the grammatical function $\mathrm{GF}_{\text {ant }}$ within some f-structure $f$, and the reflexive is embedded within $f$ to some depth defined by the path $\mathrm{GF}^{+}$. This means that the reflexive and its antecedent are either coarguments within $f$, or the reflexive is embedded inside a coargument of the antecedent (if $\mathrm{GF}^{+}$has more than one element).

In order to impose the requirement for the reflexive to be bound within its binding domain, we must place the appropriate constraints on the path $\mathrm{GF}^{+}$in (41). The English reflexive himself must be bound within the minimal complete nucleus (the minimal f-structure with a SUBJ function). This requirement is imposed by defining the path as MCNPATH:

(43) Minimal Complete Nucleus binding domain:

$$
\text { MCNPATH } \equiv \underset{\neg(\rightarrow \text { SUBJ })}{\text { GF* }^{*}} \quad \text { GF }
$$

The definition of MCNPATH in (43) contains an off-path constraint, $\neg(\rightarrow$ SUBJ): off-path constraints appear as annotations on an attribute, and allow reference to the f-structure value of the attribute $(\rightarrow)$ or to the f-structure in which the attribute appears $(\leftarrow)$. The off-path constraint $\neg(\rightarrow$ SUBJ $)$ is interpreted as constraining each non-final grammatical function GF on the path, ensuring that MCNPATH does not pass through an f-structure with a SUBJ attribute. Other binding domains involve different off-path constraints on $\mathrm{GF}^{+}$, as we will see.

Besides limiting the domain to the minimal f-structure containing a SUBJ, we must also make sure that $\mathrm{GF}_{\text {ant }}$ is constrained to range over grammatically more prominent elements within the binding domain. This prominence condition is imposed by ensuring that the f-structure value of $\mathrm{GF}_{\text {ant }}$ (the antecedent of the reflexive, labeled $a$ in 
the schematic diagram in (44)) is superior on the grammatical function hierarchy to its coargument GF which contains the reflexive, labeled $c$ in (44).

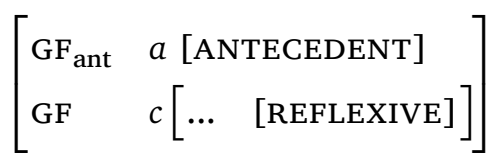

The prominence condition has never been made explicit in the LFG literature. To state it, we first define the relation SUPERIOR, which holds between arguments of the same predicate:

(45) Definition of SUPERIOR:

SUPERIOR $\left(f_{1}, f_{2}\right)$ if and only if $f_{1}$ and $f_{2}$ are arguments of the same predicate and $f_{1}$ outranks $f_{2}$ on the grammatical function hierarchy.

SUPERIOR constrains the relation between the f-structures labeled $a$ and $c$ in (44), requiring $a$ to be superior to $c$. We can now impose the appropriate prominence condition by means of the following off-path constraints on $\mathrm{GF}_{\text {ant }}$ :

(46) Off-path constraints on $\mathrm{GF}_{\text {ant }}$ encoding the superiority condition:

$$
\begin{gathered}
\mathrm{GF}_{\text {ant }} \\
\text { \%COARG }=(\leftarrow \mathrm{GF}) \\
\left(\% \operatorname{COARG} \mathrm{GF}^{*}\right)=\uparrow \\
\text { SUPERIOR } \rightarrow, \% \text { COARG })
\end{gathered}
$$

The constraints in (46) make use of a local name \%COARG to refer to a coargument of the antecedent; local names are prefixed with a percent sign '\%', and are used in order to ensure reference to the same coargument f-structure in each constraint. According to the first line, then, \%COARG is defined as an $\mathrm{f}$-structure bearing some grammatical function GF within the f-structure $\leftarrow$ : in other words, \%COARG is a coargument of the antecedent ( $c$ in (44)). According to the second constraint, \%COARG is required to (possibly improperly) contain the reflexive (since there is a possibly empty path GF* through \%COARG ending in $\uparrow$ ). According to the third constraint, the antecedent (the value of $\mathrm{GF}_{\text {ant }}, a$ in (44)) must be superior on the grammatical function hierarchy to \%COARG. For conciseness and to allow reuse of this set of con- 
straints by other lexical forms, we define the template SUPERIOR-ANT as encoding exactly this set of constraints:

(47) Definition of the template SUPERIOR-ANT:

$$
\begin{aligned}
\text { SUPERIOR-ANT } \equiv & \% \text { COARG }=(\leftarrow \text { GF }) \\
& \left(\% \text { COARG GF }{ }^{*}=\uparrow\right. \\
& \text { SUPERIOR } \rightarrow, \% \text { COARG })
\end{aligned}
$$

This allows us to succinctly state the binding conditions on himself by means of the expression in (48), with a template call @SUPERIOR-ANT to the template defined in (47):

(48) Superior f-structures in the Minimal Complete Nucleus:

(( MCNPATH $\uparrow) \quad$ GF $\left._{\text {ant }}\right)$

@SUPERIOR-ANT

The expression in (48) ranges over f-structures that constitute appropriate antecedents for himself in that they bear a superior grammatical function (as defined by the template @SUPERIOR-ANT) within the Minimal Complete Nucleus containing himself (as specified in the definition of MCNPATH).

It is easily seen from the topology of (40) that both Alan and Bertie are permissible antecedents. However, the expression in (41) picks out a single antecedent, and cannot be resolved to both at the same time. We therefore correctly predict that split antecedents are not possible with reflexives, as shown in (49).

(49) * Alan $_{i}$ told Bertie $_{j}$ about themselves $_{i+j}$

Now that we can refer to the f-structures that are syntactically suitable antecedents for a reflexive, it is possible to state the appropriate constraint on the $\mathscr{R}$ relation between the index of the reflexive and the index of its antecedent in terms of the expression in (48). This can be done by augmenting (48) with specification of the $\mathscr{R}$ relation. In (50), $\mathscr{R}$ relates the index of himself (which appears as the value of INDEX in its semantic structure $\uparrow_{\sigma}$ ) to the index of a superior f-structure within the Minimal Complete Nucleus. This constraint is specified in the lexical entry for himself.

(50) Positive binding constraint for himself:

$$
\mathscr{R}\left(\left(\uparrow_{\sigma} \text { INDEX }\right)\right)=\left(\left((\text { MCNPATH } \uparrow) \underset{\text { @SUPERIOR-ANT }}{\mathrm{GF}_{\text {ant }}}\right)_{\sigma} \text { INDEX }\right)
$$


Returning to the sentence in (39), the f-structure for Alan appears within the Minimal Complete Nucleus relative to the f-structure of the reflexive pronoun, and stands in the appropriate superiority relation to the f-structure of the reflexive pronoun. Thus, if Alan serves as antecedent to the pronoun, as shown in (40), the conditions in (43) and (50) are met, and the binding relation is permitted.

\section{PERSONAL PRONOUNS \\ AND NEGATIVE GONSTRAINTS}

We now turn to the more complex case of pronouns that are subject to negative constraints, such as English personal pronouns. As discussed in relation to example (5) above, when it comes to negative constraints we must deal with the fact that the constraint is not merely about antecedency, but about non-coreference. Antecedency implies coreference, but coreference can obtain even between elements that are not in an antecedency relation. If, therefore, we state a positive constraint defining a relation of antecedency, by implication we define a relation of coreference. But if we state a negative constraint ruling out a relation of antecedency, we do not necessarily rule out coreference.

We assume that all anaphoric indices are ultimately related to one non-anaphoric index, although the relation may not be direct. For example, consider the following sentence:

(51) Bertie said that he $\nwarrow_{1}$ thought that he $\mathrm{F}_{2}$ would win.

In (51), it may be that Bertie, $h e_{1}$ and $h e_{2}$ are all coreferent, and that $h e_{2}$ takes $h e_{1}$ as antecedent, and $h e_{1}$ takes Bertie as antecedent. In this case, $h e_{2}$ and Bertie, although coreferent, are not directly connected with one another via the $\mathscr{R}$ relation. Rather, they are related indirectly: $\mathscr{R}$ applied to the index of $h e_{2}$ finds the index of $h e_{1}$, and $\mathscr{R}$ applied to the index of $h e_{1}$ finds the index of Bertie. While antecedency corresponds to a direct relation between the index of a pronoun and the index of its antecedent, coreference corresponds to the equivalence relation we get by taking the transitive, symmetric, reflexive closure of $\mathscr{R} .{ }^{13}$ The class of discourse referents corresponding to this equivalence class of

${ }^{13}$ Observe that this applies to intended coreference only. Accidental coreference, as discussed in the binding literature, is presumably not reflected in $\mathscr{R}$. 
indices is what Kamp and Reyle $(1993,235-236)$ refer to as $[x]_{\mathrm{K}}$ in their discussion of negative constraints: the class of discourse referents identified via equality with a given discourse referent $x$ relative to a DRS $\mathrm{k}$.

We choose as a representative of the equivalence class induced by (the closure of) $\mathscr{R}$ the first, non-anaphoric index. We therefore provisionally define the function $\mathscr{R}^{*}$ as in (52); a refinement to this definition will be necessary in the analysis of plural anaphora. This definition allows us to state negative binding constraints in terms of noncoreference, as required.

(52) Definition of $\mathscr{R}^{*}$, version 1 (to be amended):

$$
\mathscr{R}^{*}(x)= \begin{cases}x & \text { if } \mathscr{R}(x) \text { is undefined } \\ \mathscr{R}^{*}(\mathscr{R}(x)) & \text { otherwise }\end{cases}
$$

$\mathscr{R}^{*}$ effectively follows the $\mathscr{R}$ path back from index to antecedent index, stopping only when it finds a non-anaphoric index: that is, an index without an antecedent. Note that $\mathscr{R}(x)$ is undefined for a nonanaphoric index, and the definition of $\mathscr{R}^{*}$ means that $\mathscr{R}^{*}(x)$ is $x$ itself, if $x$ is non-anaphoric. Choosing the first, non-anaphoric index as a representative of the coreference class is to some extent an arbitrary choice; presumably speakers do not always go back to the first mention of a new referent in a discourse. It would be possible to use instead the earliest occurrence within $n$ sentences, but we assume this is a processing issue that we can legitimately abstract away from.

As discussed above, the English personal pronouns are subject to a negative constraint that refers to the Coargument Domain of the pronoun: the pronoun may not corefer with a superior coargument. As is standard, we define the Coargument Domain in terms of the path COARGPATH:

(53) Coargument binding domain:

$$
\text { COARGPATH } \equiv \underset{\neg(\rightarrow \text { PRED })}{\text { GF* }^{*}} \quad \text { GF }
$$

Given this definition and the definition of the template @SUPERIORANT in (47), the set of f-structures which may not bind a pronoun is the set of superior f-structures in the Coargument Domain, which can be referred to in the following way: 
(54) Superior f-structures in the Coargument Domain:

(( COARGPATH $\uparrow) \quad$ GF $\left._{\text {ant }}\right)$

@SUPERIOR-ANT

The expression (COARGPATH $\uparrow$ ) refers to the f-structures in the minimal domain containing $\uparrow$, the f-structure of the pronoun, which do not properly contain an f-structure with a PRED feature. This gives us the Coargument Domain. We can then refer to superior coarguments bearing the grammatical function $\mathrm{GF}_{\mathrm{ant}}$ within this domain by imposing the same off-path constraints as in (48). These are the f-structures with which the pronoun may not corefer.

Given the definition in (52), we can now formalise the negative constraint on English personal pronouns like him and her in the following way:

(55) Negative binding constraint for English personal pronouns:

$\mathscr{R}^{*}\left(\left(\uparrow_{\sigma}\right.\right.$ INDEX $\left.)\right) \neq$

$$
\mathscr{R}^{*}\left(\left(\left((\text { COARGPATH } \uparrow) \underset{\text { @SUPERIOR-ANT }}{\text { GF }_{\text {ant }}}\right)_{\sigma} \text { INDEX }\right)\right)
$$

This constraint is specified in the lexical entry of personal pronouns such as him. It incorporates the expression in (54), which refers to superior coarguments of the personal pronoun, and it requires that the non-anaphoric index which is the antecedent (of the antecedent (of the antecedent...)) of the pronoun not be identical with any ${ }^{14}$ non-anaphoric index introduced by, or serving as antecedent (of an antecedent (of an antecedent...)) to a superior coargument of $\uparrow$. The constraint ensures that non-coreference is enforced even when the immediate antecedents of two coargument pronouns are different, by following the $\mathscr{R}$ paths back to a non-anaphoric index, and ensuring that the two paths do not lead to the same index. The use of templates such as @SUPERIOR-ANT and path definitions such as MCNPATH and COARGPATH allows us to capture commonalities in binding requirements across all anaphoric elements within and across languages.

To illustrate the effect of the constraint in (55), consider example (5), repeated in (56) with its DRS, representing the monotonic meaning of the sentence. ${ }^{15}$

\footnotetext{
${ }^{14}$ Notice that the negation scopes over the disjunction over grammatical functions in the Coargument Domain, giving universal force.

${ }^{15}$ We follow Maier (2009) in analyzing propositional attitudes as relations
} 
(56) Bertie thought that he had seen him.

\begin{tabular}{|c|c|c|}
\hline$x_{1}$ & & \\
\hline \multirow[t]{2}{*}{$\operatorname{Bertie}\left(x_{1}\right)$} & & \\
\hline & $\bar{x}_{2} \bar{x}_{3}$ & \\
\hline $\operatorname{think}\left(x_{1}\right.$ & $\operatorname{see}\left(\bar{x}_{2}, \bar{x}_{3}\right)$ & J \\
\hline
\end{tabular}

In (56), he may not serve as antecedent for him, since they are coarguments. Bertie may serve as antecedent for either he or him, but crucially may not serve as antecedent for both, since he and him may not be coreferent. The equation in (55) licenses the interpretations schematized in (57) and (58), both of which are possible, but rules out the interpretations schematized in (59) and (60), since these both involve coreference of coarguments.

(57) Bertie $_{i}$ thought that he ${ }_{j}$ had seen $\operatorname{him}_{i}$.

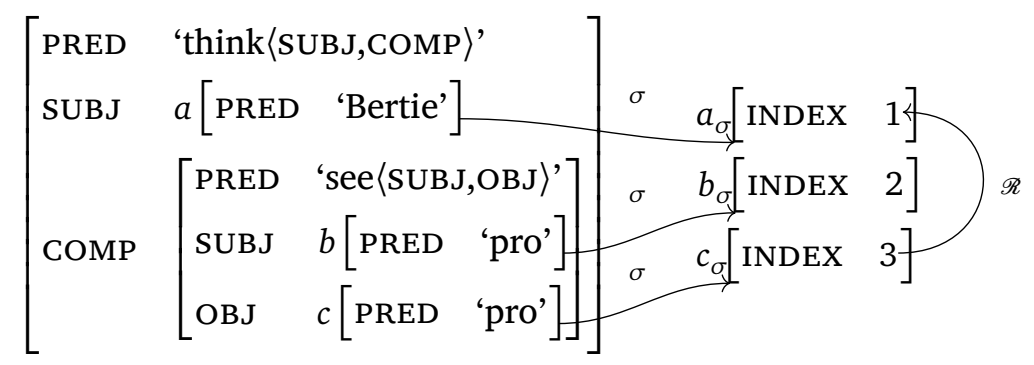

(58) Bertie $_{i}$ thought that he $e_{i}$ had seen him . $_{j}$

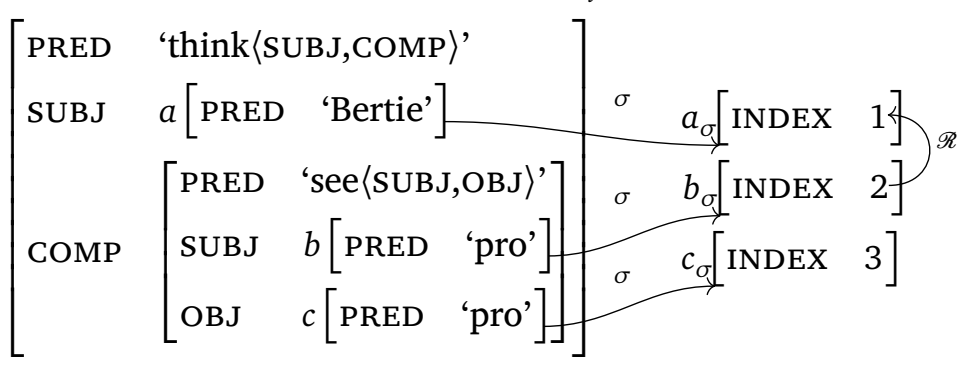

between individuals and DRSs. This would require an intensional language, but since attitudes are orthogonal to our concerns, we omit details here. 
(59) $*$ Bertie $_{i}$ thought that he ${ }_{j}$ had seen $\operatorname{him}_{j}$.

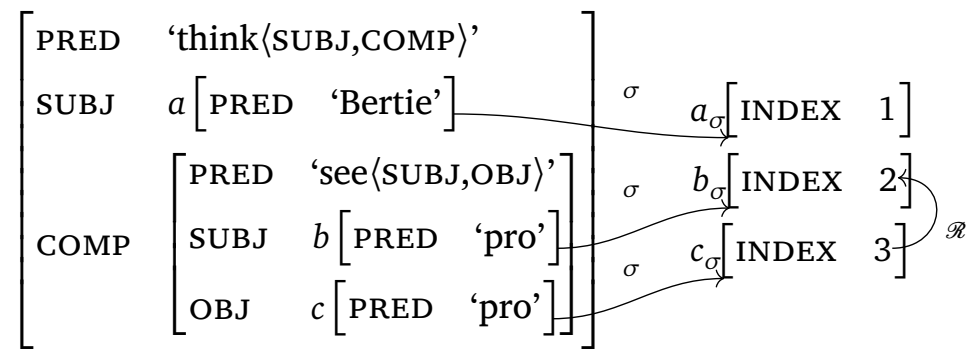

(60) * Bertie $_{i}$ thought that he $e_{i}$ had seen him . $_{i}$

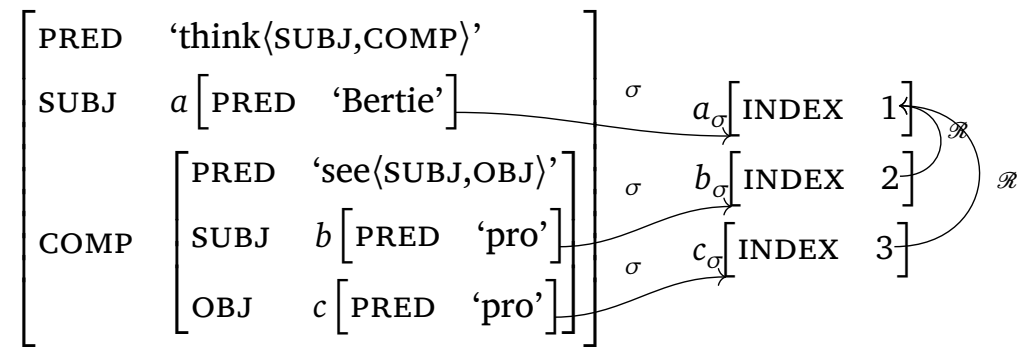

In this section, we have shown how to model the syntactic constraints imposed on the pragmatic interpretation of pronouns, using the projection architecture of LFG to constrain possible relations between indices and therefore the discourse referents that they introduce. In the next section, we show how plural pronouns complicate this picture, and we show that our model is able to handle these complications.

\section{FORMALISING PLURAL ANAPHORS}

9.1 Complex indices and complex discourse referents

As discussed above, the main complication that arises when we turn to plural pronouns is that we can no longer think purely in terms of relations between atomic indices and hence atomic discourse referents. The first fundamental change that we must make to the model presented above is to introduce a means of forming complex indices, associated with complex discourse referents, which can serve as antecedents for plural pronouns. Complex indices can be associated with 
coordinated noun phrases: for example, the index for a coordinated phrase like Alan and Bertie is a complex index formed by combining the index for Alan with the index for Bertie. We can also form complex indices by combining the indices of contextually salient discourse referents; this is necessary in the analysis of split antecedency in examples like (61), where the antecedent of them is the complex index formed from the indices of the contextually salient discourse referents for Alan and Bertie.

(61) Alan $_{i}$ told Bertie $e_{j}$ that Charlie ${ }_{k}$ admired them ${ }_{i+j}$.

On the other hand, we do not need complex indices on lexical items, as simple indices are enough to capture e.g. binding by a group noun or a plural. ${ }^{16}$ Thus, we assume that no lexical item introduces a complex discourse referent: these arise through phenomena such as coordination, split antecedence, etc.

Complex indices and discourse referents are formed by a mereological sum operator $\oplus .{ }^{17}$ For discourse referents, this is what Kamp and Reyle (1993, Chapter 4) call Summation (see also Berman and Hestvik 1997, Section 3); for indices, this is similar to what Büring (2005, Section 9.3) calls an index set, a proposal with its roots in work by Lasnik (1989a). We do not go into formal details here, but intuitively this means that we no longer have only the atomic ${ }^{18}$ indices $1,2, \ldots$ and discourse referents $x_{1}, x_{2}, \ldots$, but also complex indices $1 \oplus 2,3 \oplus 7, \ldots$ and discourse referents $x_{1} \oplus x_{2}, x_{3} \oplus x_{7}, \ldots$

The notion of mereological sum is familiar from the literature on plurals (Link 1983). Adopting precisely that theory of plurals, we can easily make sure that complex discourse referents are properly interpreted. Recall from Section 5 that it is the $v$ function that lets us move from discourse referents to their inhabitants in a given state of the discourse. To make sure that we can do the same for complex

\footnotetext{
${ }^{16}$ We assume that an example like The boys talked about him, where him is one of the boys, exemplifies Pattern B as described in Section 4. As with the examples discussed there, we assume that such examples violate no syntactic binding constraints, though they may be unacceptable for nonsyntactic reasons.

${ }^{17}$ Technically, these are distinct domains with distinct sum operators, but we simplify matters here.

${ }^{18} \mathrm{An}$ index $i$ is atomic iff there are no two distinct indices such that their mereological sum equals $i$.
} 
discourse referents, we introduce the axiom in (62), writing $\oplus^{*}$ for Link's sum operator on individuals. ${ }^{19}$

(62) Relation between complex discourse referents and their inhabitants:

$$
\forall s \forall \delta \forall \delta^{\prime} . v(s)\left(\delta \oplus \delta^{\prime}\right)=v(s)(\delta) \oplus^{*} v(s)\left(\delta^{\prime}\right)
$$

That is, in all states, the inhabitant of the complex discourse referent $\delta \oplus \delta^{\prime}$ is the sum of the inhabitants of the discourse referents $\delta$ and $\delta^{\prime}$. Note that the homomorphism from discourse referents to individuals is not (necessarily) an isomorphism, so that while non-atomic discourse referents map to non-atomic individuals, the converse is not necessarily true: as we already saw, a group noun will introduce an atomic discourse referent inhabited by a non-atomic individual.

Above, we defined $\mathscr{R}^{*}$ as a recursive version of $\mathscr{R}$, as a way of moving from indices of anaphoric expressions back to indices with no antecedent, moving perhaps through one or more indices of anaphoric expressions on the way. But this assumed that all indices are atomic. Now that we have introduced complex indices, we must update our definition of $\mathscr{R}^{*}$ accordingly. Notice that we assume that all complex indices are ultimately constructed out of atomic indices. We can therefore define a function ATOMs $(i)$ which returns the set of atomic indices that make up $i$. With this in place, we revise the definition of $\mathscr{R}^{*}$ as in (63):

(63) Definition of $\mathscr{R}^{*}$ (final; includes additional condition for complex indices):

$$
\mathscr{R}^{*}(x)= \begin{cases}x & \text { if } x \text { is atomic and } \\ & \mathscr{R}(x) \text { is undefined } \\ \mathscr{R}^{*}(\mathscr{R}(x)) & \text { if } x \text { is atomic and } \\ & \mathscr{R}(x) \text { is defined } \\ \oplus\left\{\mathscr{R}^{*}(y) \mid y \in \operatorname{ATOMS}(x)\right\} & \text { otherwise } \\ & \text { (i.e. if } x \text { is non-atomic) }\end{cases}
$$

\footnotetext{
${ }^{19}$ We can achieve the same result in a more general setting by requiring a homomorphism from the algebra of discourse referents to the algebra of individuals.
} 
In words, atomic indices are treated as before: we follow the antecedency path as far as possible. For non-atomic indices we simply apply the function to their atomic parts and take the sum of the results. To see how this works, consider the diagram in (64).

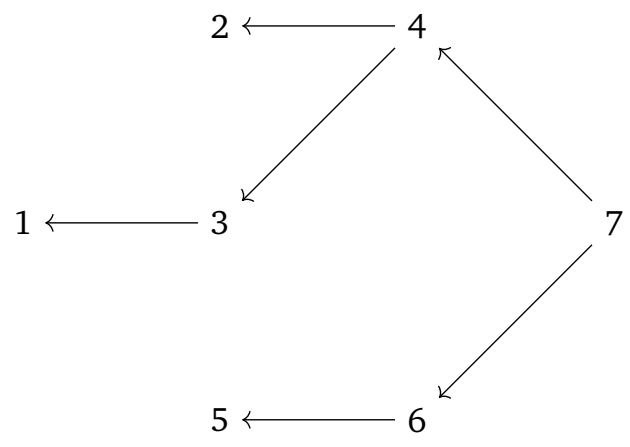

This diagram represents a situation in which there are seven indices, four of them belonging to anaphoric expressions, with the following $\mathscr{R}$ relations: $\mathscr{R}(7)=4 \oplus 6 ; \mathscr{R}(4)=2 \oplus 3 ; \mathscr{R}(6)=5 ; \mathscr{R}(3)=1$. This situation is exemplified by the following text:

(65) $\mathrm{John}_{1}$ came in and sat down. Paul 2 sat down next to him 3 , and they $_{4}$ got out their instruments. Next, George arrived, and he $_{6}$ sat down at the piano. They ${ }_{7}$ all started to sing.

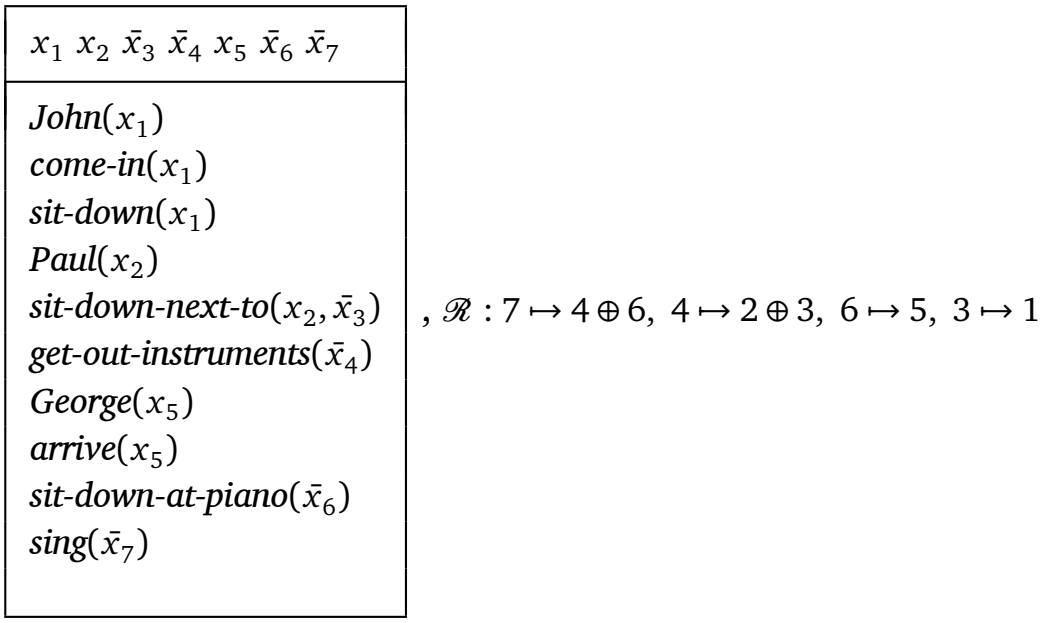

We get $\mathscr{R}^{*}(7)$ from the given $\mathscr{R}$ in the following way: By the second clause of (63), $\mathscr{R}^{*}(7)=\mathscr{R}^{*}(4 \oplus 6)$. By the third clause $\mathscr{R}^{*}(4 \oplus 6)=$ $\mathscr{R}^{*}(4) \oplus \mathscr{R}^{*}(6)$. By the two first clauses, $\mathscr{R}^{*}(6)=5$, whereas $\mathscr{R}^{*}(4)=$ 
$2 \oplus 3$, and $\mathscr{R}^{*}(3)=1$. So we get $\mathscr{R}^{*}(7)=\mathscr{R}^{*}(4 \oplus 6)=1 \oplus 2 \oplus 5$ (since mereological sum is associative).

\section{3 \\ Reflexives with plural antecedents}

In the case of the positive constraint on English reflexive pronouns, plurality has little effect on the generalisations. A reflexive pronoun must be coreferent with an antecedent in its Minimal Complete Nucleus, whether it is singular or plural. Partial coreference is not possible: for example, one cannot say the following, to mean that Bertie likes himself and one or more others:

(66) *Bertie likes themselves.

Likewise, one cannot say the following, to mean that Alan and Bertie like one of either Alan or Bertie:

(67) *Alan and Bertie like himself.

A reflexive pronoun must take a single (possibly complex, but not split) index as its antecedent. Given our analysis of complex indices in terms of mereological sums, the plural case falls directly out of equation (50), as shown in (68)-(69). Note that the f-structure for a coordinate structure like Alan and Bertie is a set (labeled z), and the conjuncts are elements of the set (labeled $a$ and $b$ ).

(68) * $\operatorname{Alan}_{i}$ and Bertie $_{j}$ like himself $_{j}$.

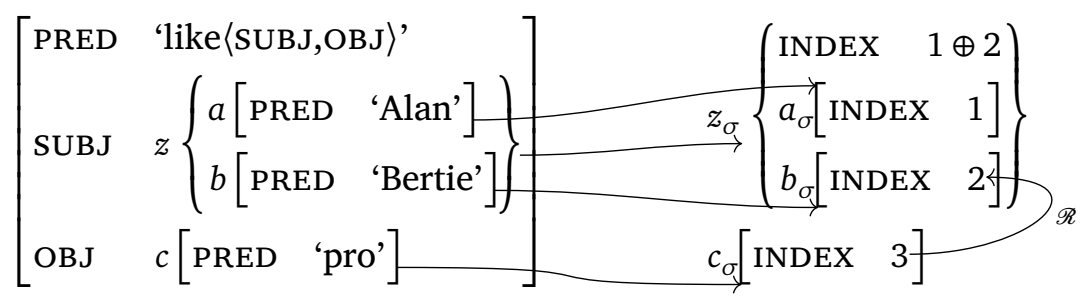

(69) $\operatorname{Alan}_{i}$ and Bertie $_{j}$ like themselves ${ }_{i+j}$.

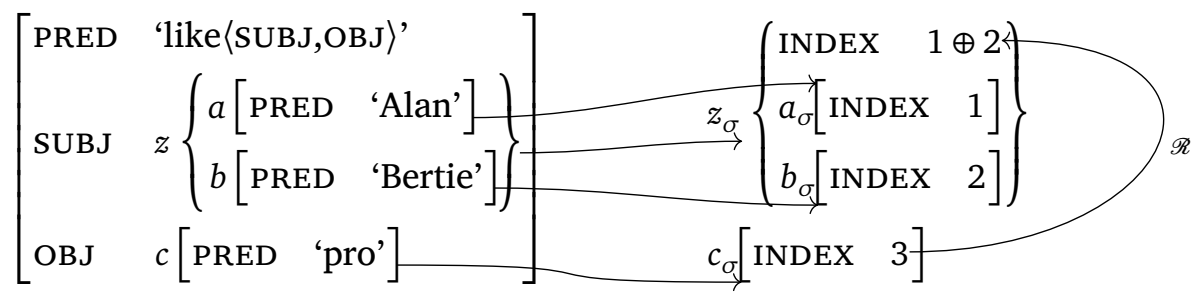


The f-structure $z$ is the only f-commanding GF in the Minimal Complete Nucleus relative to $c$. The only licit antecedent is therefore $\left(z_{\sigma}\right.$ INDEX), which is $1 \oplus 2$. The f-structures for Alan and Bertie are not syntactically suitable antecedents - in particular, they do not $\mathrm{f}$ command the pronoun - and so their indices 1 and 2 are not individually available as antecedents.

Pronouns with split antecedents

With (63) in place, it is unproblematic to account for examples like (61), repeated here, making use of the negative constraint in (55).

(70) Alan $_{1}$ told Bertie ${ }_{2}$ that Charlie ${ }_{3}$ admired them . $_{4}$

Let us check that the resolution $\mathscr{R}^{*}(4)=1 \oplus 2$ is valid. If we assume that these are the first occurrences of Alan, Bertie, and Charlie in the discourse, we get $\mathscr{R}^{*}(1)=1, \mathscr{R}^{*}(2)=2, \mathscr{R}^{*}(3)=3$, and $\mathscr{R}^{*}(4)=1 \oplus 2$. By (55), $\mathscr{R}^{*}(4)$ must be different from $\mathscr{R}^{*}$ applied to any index projected from a superior element in its binding domain, which is the Coargument Domain. The only superior coargument is Charlie, so $\mathscr{R}^{*}(4)$ must be different from $\mathscr{R}^{*}(3)$, which it is.

9.5

\section{Comparison with other approaches}

To our knowledge, Berman and Hestvik (1997) is the most recent attempt to deal with the binding patterns of plural pronouns. Besides offering a more precise formalization, Berman and Hestvik (1997) also discuss and improve upon certain aspects of Lasnik (1989a), Seeley (1993), and Fiengo and May (1994). Therefore, we only compare our approach to Berman and Hestvik (1997) here.

The main empirical difference between our approach and that of Berman and Hestvik (1997) concerns Principle B effects in ditransitives. They claim that it is ungrammatical for a pronoun to corefer with the sum of its superior coarguments (Pattern D above) and set their theory up accordingly. Our theory instead predicts that a pronoun must be non-coreferent with each of its superior coarguments, meaning that Pattern D is grammatical.

As we noted in our discussion of Pattern D in Section 4, the empirical evidence is unclear. Note that both approaches make the same predictions about standard, monotransitive cases like John likes him, 
because the sum of superior coarguments of the pronoun in such cases just is the single superior coargument.

The theories also differ in the predictions they make about examples where a pronoun corefers with one of several superior coarguments. Such examples are in fact unacceptable, and are judged as such by Berman and Hestvik themselves.

(71) * $\mathrm{John}_{i}$ told Mary ${ }_{j}$ about her ${ }_{j} /$ him $_{i}$. (Berman and Hestvik 1997, 25)

This pattern is incorrectly classified as grammatical by the restriction on coreference proposed by Berman and Hestvik (1997, 22): "the restriction on CR.PRO [coreference resolution of pronouns] is simply that no DRS-equivalent of a potential resolving discourse referent for a pronoun may be identical to the set of discourse referents that c-command the pronoun within its binding domain". Berman and Hestvik appear not to have noticed that this runs counter to their judgements about examples like (71). Their theory could probably be amended by making the generalization (and the corresponding formalization) disjunctive ("or with a single c-commanding discourse referent"). Similarly, should further empirical investigation reveal that Pattern $\mathrm{D}$ is indeed ungrammatical, our theory could be amended with the extra constraint in (72).

(72) Additional negative condition for plural pronouns, requiring noncoreference with the sum of the coarguments:

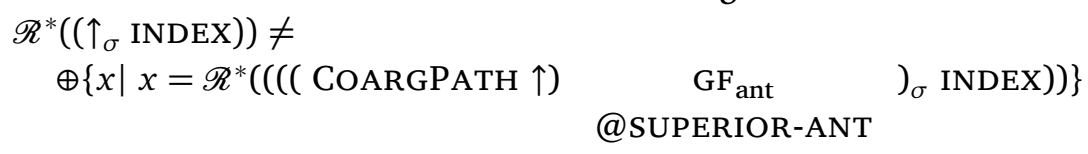

However, at this stage, we do not see any way of ruling out Pattern D and the other illicit binding patterns for pronouns by means of a single, nondisjunctive constraint. That is, should Pattern D turn out to be ungrammatical, it seems that the negative binding constraints on (plural) pronouns would have to be essentially disjunctive.

\section{GONGLUSION}

The interaction of syntactic and pragmatic constraints on pronominal reference provides a challenge for any model of grammar. Our 
approach offers an integrated account in which syntactic and pragmatic factors jointly constrain binding possibilities. In our model, binding theory is stated in terms of syntactic constraints on pragmatic anaphora resolution. The modular grammatical architecture of LFG provides a natural setting for this integration, with its clean separation of syntactic, semantic, and pragmatic components of the grammar. In this, our analysis represents a step forward from the most recent indepth work on binding of plural anaphora, the work of Berman and Hestvik (1997), who present an approach involving rewriting of Government and Binding-style phrase structure trees into DRSs. We also provide for the first time a full formal treatment of coreference relations and negative binding constraints in an LFG setting. Our analysis crucially relies on the Partial Compositional Discourse Representation Theory of Haug (2014b), with its explicit separation between the semantic and pragmatic contributions of anaphoric elements.

Regarding the empirical data for plural anaphora, we have identified four possible patterns of inclusion between the index of a pronoun and its antecedent, some of which have been subject to varying grammaticality judgements in previous literature. Our formal analysis classifies these patterns as syntactically wellformed, and we anticipate that further research will uncover other factors, such as lexical and contextual factors, to explain unacceptable instances.

Further potential for our analysis includes its extension to modelling constraints on resumptive pronouns (Asudeh 2011, 2012) and null pronouns e.g. in anaphoric control constructions; PCDRT has already been extended to deal with the anaphoric relations inherent in partial control constructions (Haug 2014a; see also Haug 2013 and Belyaev and Haug 2014).

\section{AGKNOWLEDGMENTS}

We are grateful to the audience at LFG17 in Konstanz for comments on an early version of this paper, particularly to Ash Asudeh and Geoff Pullum. We are also grateful to Jamie Findlay, Adam Przepiórkowski, and three anonymous JLM reviewers for comments on a later version. This article was finished while the two first authors were on sabbatical at the Centre for Advanced Study at the Norwegian Academy of Science and Letters. We gratefully acknowledge their support. 


\section{REFERENGES}

Ash ASUDEH (2011), Towards a unified theory of resumption, in Alain ROUVERET, editor, Resumptive pronouns at the interfaces, pp. 121-187, Benjamins, Amsterdam.

Ash ASUDEH (2012), The logic of pronominal resumption, Oxford University Press, Oxford.

Ash AsudeH and Gianluca Giorgolo (2012), Flexible composition for optional and derived arguments, in Miriam BUTT and Tracy Holloway KING, editors, Proceedings of the LFG12 Conference, pp. 64-84, CSLI Publications, Stanford, CA.

Ash Asudeh, Gianluca Giorgolo, and Ida Toivonen (2014), Meaning and valency, in Miriam BUTT and Tracy Holloway KING, editors, Proceedings of the LFG14 Conference, pp. 68-88, CSLI Publications, Stanford, CA.

David I. BEAVER (1992), The kinematics of presupposition, in Paul DEKKER and Martin SтоскноF, editors, Proceedings of the Eighth Amsterdam Colloquium, ILLC, University of Amsterdam.

David I. BEAVER (2002), Presupposition projection in DRT: a critical

assessment, in David I. BEAVER, editor, The construction of meaning, pp. 23-43, CSLI Publications, Standford, CA.

Oleg I. BelyaeV and Dag T. T. HAug (2014), Pronominal coreference in Ossetic correlatives and the syntax-semantics interface, in Miriam BUTT and Tracy Holloway KING, editors, Proceedings of the LFG14 Conference, pp. 89-109, CSLI Publications, Stanford, CA.

Stephen BERMAN and Arild HESTVIK (1997), Split antecedents, noncoreference, and DRT, in Hans BENNIS, Pierre PICA, and Johan ROORYCK, editors, Atomism and Binding, pp. 1-29, Foris, Dordrecht.

Tina BöGEL, Miriam ButT, Ronald M. KAPLAN, Tracy Holloway KING, and John T. MAXWELL III (2009), Prosodic phonology in LFG: a new proposal, in Miriam BUTT and Tracy Holloway KING, editors, Proceedings of the LFG09 Conference, pp. 146-166, CSLI Publications, Stanford, CA.

Joan Bresnan, Ash Asudeh, Ida Toivonen, and Stephen Wechsler (2016), Lexical-functional syntax, Wiley-Blackwell, Oxford, second edition. First edition by Joan Bresnan, 2001, Blackwell.

Joan Bresnan, Per-Kristian Halvorsen, and Joan Maling (1985), Logophoricity and bound anaphors, unpublished manuscript, Department of Linguistics, Stanford University.

Daniel BÜRING (2005), Binding theory, Cambridge University Press, Cambridge. Gennaro CHIERCHIA (2004), Scalar implicatures, polarity phenomena, and the syntax/pragmatics interface, in Adriana BELLETTI, editor, Structures and 
beyond: the cartography of syntax structures, vol. 3, pp. 39-103, Oxford University Press, Oxford.

Noam CHOMSKY (1973), Conditions on transformations, in Stephen ANDERSON and Paul KIPARSKY, editors, A Festschrift for Morris Halle, Holt, Rinehart \& Winston, New York.

Noam CHомsкy (1981), Lectures on Government and Binding: the Pisa lectures, Foris, Dordrecht.

Mary DALRYMPLE (1993), The syntax of anaphoric binding, CSLI Publications, Stanford, CA.

Mary DALRYMPLE (2001), Lexical Functional Grammar, Academic Press, San Diego, CA.

Mary DALRYMPLE and Louise MYCOCK (2011), The prosody-semantics interface, in Miriam BUTT and Tracy Holloway KING, editors, Proceedings of the LFG11 Conference, pp. 173-193, CSLI Publications, Stanford, CA.

Mary DALRYMPLE and Irina NiKolAEVA (2006), Syntax of natural and accidental coordination: evidence from agreement, Language, 82(4):824-849.

Mary DALRYMPLE and Irina NiKOlAEVA (2011), Objects and information structure, Cambridge University Press, Cambridge.

Yehuda N. FALK (2001), Lexical-Functional Grammar: an introduction to parallel constraint-based syntax, CSLI Publications, Stanford, CA.

Robert FIENGO and Robert MAY (1994), Indices and identity, Linguistic Inquiry Monographs, The MIT Press, Cambridge, MA.

Gianluca Giorgolo and Ash AsUdeH (2011), Multimodal communication in LFG: gestures and the correspondence architecture, in Miriam BUTT and Tracy Holloway KING, editors, Proceedings of the LFG11 Conference, pp. 257-277, CSLI Publications, Stanford, CA.

Dag T. T. HAUG (2013), Partial control and the semantics of anaphoric control in LFG, in Miriam BUTT and Tracy Holloway KING, editors, Proceedings of the LFG13 Conference, pp. 274-294, CSLI Publications, Stanford, CA.

Dag T. T. HAUG (2014a), The anaphoric semantics of partial control, Proceedings of SALT, 24:213-233.

Dag T. T. HAUG (2014b), Partial dynamic semantics for anaphora: compositionality without syntactic coindexation, Journal of Semantics, 31(4):457-511, first published online August 24, 2013. DOI:

10.1093/jos/fft008.

Irene R. HEIM (1982), The semantics of definite and indefinite noun phrases, Ph.D. thesis, University of Massachusetts, Amherst.

James Higginвотнам (1983), Logical form, binding, and nominals, Linguistic Inquiry, 14(3):395-420. 
Ray JACKENDOFF (1992), Mme. Tussaud meets the binding theory, Natural Language and Linguistic Theory, 10(1):1-32.

Hans KAMP and Uwe REYLE (1993), From discourse to logic, Kluwer Academic Publishers, Dordrecht.

Hans KAMP, Josef VAN GENABITH, and Uwe REYLE (2011), Discourse Representation Theory, in Dov M. GABBAY and Franz GÜNTHNER, editors, Handbook of philosophical logic, pp. 125-394, Springer, Dordrecht, second edition.

Ronald M. KAPLAN (1989), The formal architecture of Lexical-Functional Grammar, in Chu-Ren HUANG and Keh-Jiann CHEN, editors, ROCLING II: Proceedings of the Computational Linguistics Conference, pp. 3-18, The Association for Computational Linguistics and Chinese Language Processing (ACLCLP), Tapei, also published in Journal of Information Science and Engineering 5 (1989), pp. 305-322, and in Formal issues in Lexical-Functional Grammar, ed. Mary Dalrymple, Ronald M. Kaplan, John T. Maxwell III and Annie Zaenen, CSLI Publications, 1995, pp. 7-27.

Ronald M. KAPLAN and Joan BRESNAN (1982), Lexical-Functional Grammar: a formal system for grammatical representation, in Joan BRESNAN, editor, The mental representation of grammatical relations, pp. 173-281, MIT Press, Cambridge, MA.

Edward L. KEENAN and Bernard COMRIE (1977), Noun phrase accessibility and universal grammar, Linguistic Inquiry, 8(1):63-99.

Paul KIPARSKY (2002), Disjoint reference and the typology of pronouns, in Ingrid KAUFMANN and Barbara STIEBELS, editors, More than words: a Festschrift for Dieter Wunderlich, pp. 179-226, Akademie Verlag, Berlin.

Miltiadis KоккоNIDIS (2008), First-order Glue, Journal of Logic, Language and Information, 17:43-68.

Howard LASNIK (1989a), On the necessity of binding conditions, in Essays on anaphora, Kluwer, Dordrecht.

Howard LASNIK (1989b), On two recent treatments of disjoint reference, in Essays on anaphora, Kluwer, Dordrecht.

Howard LASNIK (1989c), Remarks on coreference, in Essays on anaphora, Kluwer, Dordrecht.

Howard LASNIK (1989d), A selective history of modern binding theory, in Essays on anaphora, Kluwer, Dordrecht.

Godehard LINK (1983), The logical analysis of plurals and mass terms: a lattice-theoretical approach, in Rainer BÄUERLE, Chirstoph SCHWARZE, and Arnim VON STECHOW, editors, Meaning, use and the interpretation of language, pp. 303-323, de Gruyter, Berlin. 
Emar MAIER (2009), Presupposing acquaintance: a unified semantics for de dicto, de re and de se belief reports, Linguistics and Philosophy, 32:429-474.

Reinhard Muskens (1996), Combining Montague Semantics and Discourse Representation, Linguistics and Philosophy, 19:143-186.

Louise MYCOCK and John J. LOWE (2013), The prosodic marking of discourse functions, in Miriam BuTT and Tracy Holloway KING, editors, Proceedings of the LFG13 Conference, pp. 440-460, CSLI Publications, Stanford, CA.

Rick NouWEN (2003), Plural pronominal anaphora in context, Ph.D. thesis, Utrecht Institute of Linguistics OTS.

Karen PARK (2012), The selective properties of verbs in reflexive constructions, D.Phil. thesis, University of Oxford.

Tanya REINHART and Eric REULAND (1993), Reflexivity, Linguistic Inquiry, 28:178-187.

T. Daniel SEELEY (1993), Binding plural pronominals, in Katherine BEALS, Gina Cooke, David Kathman, Sotaro KitA, Karl-Erik McCullough, and David TESTEN, editors, CLS29: papers from the $29^{\text {th }}$ regional meeting of the Chicago Linguistic Society, vol. II, pp. 305-317, University of Chicago, Chicago.

Thomas WASOW (1972), Anaphoric relations in English, Ph.D. thesis, Massachusetts Institute of Technology.

This work is licensed under the Creative Commons Attribution 3.0 Unported License. http://creativecommons.org/licenses/by/3.0/ 